\title{
RECENT ADVANCES IN THE THEORY OF THE HYDROGEN LAMB SHIFT
}

\author{
H. GROTCH \\ 104 Davey Laboratory, Department of Physics, Penn State University \\ University Park, PA 16802, USA
}

\section{Dedicated to Prof. Iwo Biatynicki-Birula on the occasion of his 60th birthday}

The theory of the $2 S_{\frac{1}{2}}-2 P_{\frac{1}{2}}$ Lamb shift of hydrogen is discussed and compared to experiment. Recent corrections from radiative recoil of order $\alpha(Z \alpha)^{5} m^{2} / M$ and pure recoil of order $(Z \alpha)^{6} \mathrm{~m}^{2} / M$ are reviewed. Contributions of order $\alpha^{2}(Z \alpha)^{5} m$ have been calculated from certain classes of diagrams. These are described while other as yet uncalculated terms are mentioned. The largest error in the theory is the uncertain measured value of the proton electromagnetic radius.

PACS numbers: 12.20.Ds, 12.20.Fv, 11.10.St

\section{Introduction}

The Lamb shift of hydrogen has provided a test of quantum electrodynamics and of bound state methods in quantum field theory. The original measurement of the $2 S_{\frac{1}{2}}-2 P_{\frac{1}{2}}$ splitting carried out in 1947 by Lamb and Retherford [1], resulted in a separation of approximately $1000 \mathrm{MHz}$.

Experimental results were presented at the Conference on the Foundations of Quantum Mechanics held at Shelter Island on June 1-3, 1947. Shortly after that conference Hans Bethe proposed an explanation for the Lamb shift in which the energy separation was based on the interaction of the bound electron with the quantized radiation field [2]. Schwinger and Weisskopf [3] and earlier Oppenheimer [4] had already made the suggestion that the electron should be coupled to this field. However, they found that this coupling produced a linearly divergent energy shift. Bethe was able to show that this divergence could be absorbed in an electromagnetic mass shift and that the residual energy shift, while still logarithmically divergent, gave approximately the correct result if the divergence was cut off at the electron mass.

The important research of Lamb and Bethe was followed by more than four decades of experimental and theoretical refinement. Currently, the experimental precision is approaching 1 part per million (about $1 \mathrm{kHz}$ ) for the $2 S-2 P$ separation 
of hydrogen [5, 6]. There has been extensive research on high $Z$ Lamb shifts [7], as well as on the bound states of $\mathrm{He}^{+}[8]$. These also give high precision results. We will not review these areas since this paper is devoted primarily to hydrogen.

In Sec. 2 we present the most recent theoretical results for the Lamb shift along with a discussion of the various components of the formula. In Sec. 3 we discuss experimental results and compare them to the theory. In Secs. 4, 5, and 6, we describe work on radiative recoil corrections of order $\alpha(Z \alpha)^{5} \mathrm{~m}^{2} / M$, pure recoil of order $(Z \alpha)^{6} \mathrm{~m}^{2} / M$, and nonrecoil of order $\alpha^{2}(Z \alpha)^{5} \mathrm{~m}$. In the concluding Sec. 7, we discuss remaining uncertainties, including uncalculated parts of the theory.

\section{Theoretical expression for the Lamb shift $\left(2 S_{\frac{1}{2}}-2 P_{\frac{1}{2}}\right)$}

At present the expression for the Lamb shift is given below with numerical contribution of each piece in megahertz $(\mathrm{MHz})$ given to the right:

$$
\begin{array}{l|c}
\delta E=\Delta E_{2 S^{\frac{1}{2}}}-\Delta E_{2 P_{\frac{1}{2}}} & \mathrm{MHz} \\
=\frac{\alpha(Z \alpha)^{4} m}{6 \pi}\left\{( \frac { \mu } { m } ) ^ { 3 } \left[\frac{1}{8} \frac{m}{\mu}+\ln (Z \alpha)^{-2}-2.207909\right.\right. & 1050.560 \\
+Z \alpha \pi\left(\frac{427}{128}-\frac{3}{2} \ln 2\right) & 7.129 \\
+(Z \alpha)^{2}\left(-\frac{3}{4} \ln ^{2}(Z \alpha)^{-2}+\left(4 \ln 2+\frac{55}{48}\right) \ln (Z \alpha)^{-2}\right) & -0.246 \\
+(Z \alpha)^{2}(-23.42 \pm 0.2) & -0.169 \\
\left.+\alpha\left(\frac{0.323}{\pi}\right)\right] & 0.101 \\
\left.+\frac{Z m}{M}\left[\frac{1}{4} \ln (Z \alpha)^{-2}+2.39977\right]\right\} & 0.359 \\
+\frac{1}{12}(Z \alpha)^{4} m \cdot m^{2}\left\langle r_{\mathrm{p}}^{2}\right\rangle & 0.145 \text { if } r_{\mathrm{p}}=0.862(12) \mathrm{fm} \\
& \left(0.127 \text { if } r_{\mathrm{p}}=0.805(11) \mathrm{fm}\right) \\
-\frac{1}{48}(Z \alpha)^{4} m \cdot \frac{m^{2}}{M^{2}} & -0.0022 \\
+\frac{\alpha(Z \alpha)^{5} m^{2}}{8 M}\left[\left(\frac{35}{4} \ln 2-\frac{7333}{960}\right)-0.415\right] & -0.0025 \\
+\frac{(Z \alpha)^{5} m^{2}}{6 \pi M} \cdot\left(\frac{3}{4} \pi Z \alpha\right)\left[\frac{5}{2}+\ln (2 Z \alpha)^{-1}-4.25\right] & 0.0032 \\
+\frac{\alpha^{2}(Z \alpha)^{5} m}{8 \pi}[-0.06+0.51+0.61-0.07 & 0.0053 \\
+e+f] & ? \\
\cline { 2 - 2 } & 1057.883 \text { if } r_{\mathrm{p}}=0.862(12) \mathrm{fm} \\
& 1057.865 \text { if } r_{\mathrm{p}}=0.805(11) \mathrm{fm}
\end{array}
$$

This expression has many contributing terms. The terms which have been computed numerically introduce negligible errors (less than $1 \mathrm{kHz}$ ). We will now describe the different pieces. The leading term, of order $\alpha(Z \alpha)^{4} m$, gives $1050.56 \mathrm{MHz}$ [9]. The bulk of this arises from the leading piece of the bound electron self-energy while part comes from vacuum polarization. The expression given includes reduced mass corrections. To properly obtain these mass corrections it is useful to include the proton kinetic energy and the transverse photon exchange potential in the initial Dirac equation. A systematic analysis of these mass corrections is given in Ref. [10].

The leading relativistic corrections of order $\alpha(Z \alpha)^{5} m$ have been evaluated by many authors [11]. It was originally expected that these terms would be modified by the factor $(\mu / m)^{3}$ which occurs whenever a result is proportional to the square of the wave function at the origin. However, as shown in Ref. [10], this factor does not always appear since there are radiative recoil contributions which are also present. Nevertheless, the leading relativistic correction, including a factor 
of $(\mu / m)^{3}$ which gives part of the mass correction, results in the contribution $7.129 \mathrm{MHz}$.

Next there are yet smaller relativistic corrections of $\alpha(Z \alpha)^{6} m$ which are multiplied by factors of $\ln ^{2}(Z \alpha)^{-2}, \ln (Z \alpha)^{-2}$, and 1 . The coefficients of the logarithmic pieces are known analytically [12] while the nonlogarithmic piece has been estimated and also later calculated numerically [13]. The numerical result quoted above is given by Wijngaarden et al. [14]. Recently there also has been a separate calculation carried out by Pachucki [15]. Respective contributions of $-0.246 \mathrm{MHz}$ and $-0.169 \mathrm{MHz}$ come from the logarithmic and nonlogarithmic pieces.

The contribution $\alpha^{2}(Z \alpha)^{4} m$ gives $0.101 \mathrm{MHz}$ and arises from contributions to the slope of the Dirac form factor, as well as anomalous magnetic moment terms. It also includes fourth order vacuum polarization on a single Coulomb line [16].

The terms which give $0.359 \mathrm{MHz}$ are of order $(Z \alpha)^{5} \mathrm{~m}^{2} / M$ and are of the same order as reduced mass contributions to the leading radiative level shift [17]. These terms, known as pure recoil terms, have an entirely different origin. They come from double Coulomb interactions (ladder plus crossed graphs) not already contained in the second Born approximation of the Coulomb interaction, from double transverse photon exchange, and from the effects of single transverse exchange not already present in the approximation based on use of the Breit potential.

Next there is the very important contribution $0.145 \mathrm{MHz}$ or $0.127 \mathrm{MHz}$ which comes from the deviation of the proton from a point particle [18]. The modification of the Coulomb potential due to the finite extension of the proton perturbs the $S$ state but not $P$ state energy since the $S$ state has a finite wave function near the origin, while the $P$ state does not. We have quoted results based on two measurements of the proton radius $[19,20]$. The uncertainty in the proton radius produces the largest known source of error for the hydrogen Lamb shift. We will return to further discuss this in Sec. 7.

The quadratic recoil correction which follows gives $-0.0022 \mathrm{MHz}$. This was worked out many years ago [21] and discussed more recently [22]. It arises from the Dirac equation with a potential which includes both the Coulomb and Breit interactions.

The next three corrections respectively give radiative recoil [10], additional pure recoil [23,24], and higher order radiative effects which involve double Coulomb exchange [25-27]. The first two terms above give $-0.0025 \mathrm{MHz}$ and $0.0032 \mathrm{MHz}$, respectively while the last is incomplete but gives $0.0057 \mathrm{MHz}$ from the diagrams calculated to date. A discussion of this work will be presented in Secs. 4, 5, and 6 .

\section{Experimental results and comparison with theory}

The most recent experimental results for the $2 S_{\frac{1}{2}}-2 P_{\frac{1}{2}}$ Lamb shift are the following:

1057.845(9) MHz (Lundeen and Pipkin [5]) and

1057.851(2) MHz (Palchikov, Sokolov, and Yakovlev [6]).

These experiments were an improvement of early work. Lundeen and Pipkin passed a beam of $2 S$ atoms through an $r f$ field. In a mixing region there was 
a static electric field which mixed $2 S$ and $2 P$ states. This mixing then caused Lyman $\alpha$-radiation to be emitted. If the $r f$ field is resonant with $2 P_{\frac{1}{2}}$, transitions out of $2 S_{\frac{1}{2}}$ occur, thus depleting the $2 S$ beam and consequently the subsequent $\alpha$-radiation. In this experiment Lundeen and Pipkin use two spatially separated $r f$ fields to quench the $2 S$ state. They found that this approach could be used to narrow significantly the line width.

Palchikov, Sokolov, and Yakovlev used a somewhat different approach. Atoms in the $2 S_{\frac{1}{2}}$ state $(F=0)$ passed through two short regions separated by $L$. In these regions there was a longitudinal electric field. Radiation from atoms in $2 P_{\frac{1}{2}}$ state was detected by a photodetector when they exit the second electric field region. The signal detected is the difference of the number of atoms in the $2 P_{\frac{1}{2}}$ state when the $\boldsymbol{E}$ field was reversed in the second region. The signal was measured as a function of $L$ and a fit was made to determine the Lamb shift as a function of beam velocity. A separate experiment was used to determine the beam velocity by observing the decay of the Lyman $\alpha$-radiation after the metastable atoms passed through the first electric field region. This was essentially a measurement of the Lamb shift in terms of the lifetime of the $2 P_{\frac{1}{2}}$ state.

As mentioned previously, the largest uncertainty in the theory of the Lamb shift is due to our lack of knowledge of the proton radius. Prior to the experiment reported in Ref. [19] the value used for the radius was $0.805 \mathrm{fm}$ [20]. This older value gives a smaller result for the Lamb shift theory by $18 \mathrm{kHz}$, providing better agreement with experiment. In recent years there has been a number of theoretical developments which have altered the theory. There was a $4 \mathrm{kHz}$ increase due to a more precise numerical calculation of the $\alpha(Z \alpha)^{6} \mathrm{~m}$ piece [14], a $-2.2 \mathrm{kHz}$ change due to quadratic recoil [21, 22], a $-2.5 \mathrm{kHz}$ effect from radiative recoil [10], a $3.2 \mathrm{kHz}$ contribution from pure recoil [23, 24], and thus far a $5.3 \mathrm{kHz}$ addition from two-loop nonrecoil graphs $[25,26,27]$. These changes currently add up to an $8.2 \mathrm{kHz}$ increase. At present the theoretical value of $1057.883 \mathrm{MHz}$ disagrees with experiment. However, we wish to stress that the theory is incomplete since additional $\alpha^{2}(Z \alpha)^{5} m$ must be calculated. In addition the error arising from the uncertain value of the proton radius is quite large.

\section{Radiative recoil corrections of order $\alpha(Z \alpha)^{5} \mathrm{~m}^{2} / M$}

Many years ago Grotch and Yennie [28] proposed and solved a modified Dirac equation for hydrogen

$$
\left[\boldsymbol{\alpha} \cdot(\boldsymbol{p}-e \boldsymbol{A})+\beta m+V+\frac{p^{2}}{2 M}\right]|n\rangle=E_{n}|n\rangle
$$

or

$$
(\not I-m)|n\rangle=0
$$

with $\Pi_{0}=E_{n}-V-\frac{p^{2}}{2 M}$ and $\boldsymbol{\Pi}=\boldsymbol{p}-e A$, where $V$ is the Coulomb potential and $-e \boldsymbol{\alpha} \cdot \boldsymbol{A}$ is the convection interaction due to the motion of the nucleus. The nuclear kinetic energy is also included in the Hamiltonian. When $M$ approaches infinity we obtain the static limit. 
The leading contribution to the Lamb shift, which arises from the electron self-energy in an external Coulomb field is given by the expression

$$
\Delta E_{n}=\frac{\alpha}{4 \pi^{3}} \int \frac{\mathrm{d}^{4} k / \mathrm{i}}{k^{2}+\mathrm{i} \epsilon}\left\langle n\left|\gamma_{\mu} \frac{1}{\not 1-\not k-m+\mathrm{i} \epsilon} \gamma^{\mu}\right| n\right\rangle-\delta m\langle n \mid n\rangle
$$

and is graphically depicted by Fig. 1 . When recoil is ignored $\Pi_{0}=E_{n}-V$ and $\Pi=\boldsymbol{p}$. A part of the recoil corrections may be calculated by using Eq. (3) above

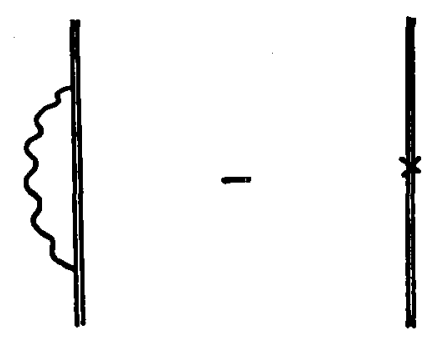

Fig. 1. Electron self-energy in a Coulomb field.

but with $\Pi_{\mu}$ given by the mass-corrected expression. We call this approach the external field approximation (EFA). Figure 1 now contains on the external and internal lines ladder Coulomb and convection interactions. In addition, the nonrelativistic nuclear kinetic energy is included. The Erickson-Yennie [11] approach to the evaluation of $\Delta E_{n}$ was carried out in Ref. [10] with the following results:

$$
\begin{aligned}
\Delta E_{n}^{(4)}= & \frac{4 \alpha(Z \alpha)^{4} m}{3 \pi n^{3}}\left\{\left(\frac{\mu_{r}}{m}\right)^{3}\left[\frac{3}{8}+\frac{11}{24}+\ln (Z \alpha)^{-2}+\ln \left(1+\frac{m}{M}\right)\right] \delta_{\ell 0}\right. \\
& \left.+\frac{3}{8}\left(\frac{\mu_{r}}{m}\right)^{2} C_{\ell j} \delta_{\ell 1}+\left(\frac{\mu_{r}}{m}\right)^{3} \ln \frac{\mu_{r}(Z \alpha)^{2}}{2 \Delta \epsilon_{n}}\right\}, \\
\Delta E_{n}^{(5)}= & \frac{4 \alpha(Z \alpha)^{5} m}{n^{3}}\left(\frac{\mu_{r}}{m}\right)^{3}\left(1+\frac{11}{128}-\frac{1}{2} \ln 2\right) \delta_{\ell 0} \\
& +\frac{\alpha(Z \alpha)^{5}}{n^{3}} \frac{m^{2}}{M}\left(\frac{35}{4} \ln 2-\frac{7333}{960}\right) \delta_{\ell 0},
\end{aligned}
$$

where $\mu_{r}=m M /(m+M)$ is the reduced mass, $C_{1, \frac{1}{2}}=-1 / 3, C_{1, \frac{3}{2}}=1 / 6$ and $\Delta \epsilon_{n}$ is the average excitation energy. The second term of $\Delta E_{n}^{(5)}$ provides a correction beyond reduced mass modifications presented earlier in the literature. It contributes a $-2 \mathrm{kIIz}$ correction to the $n=2$ hydrogen Lamb shift.

The approach based on Eq. (4) with state vectors $|n\rangle$ which satisfy Eq. (2) is systematic but Eq. (4) is an approximation which excludes those Feynman graphs which do not follow from this ladder approach. To obtain Eq. (4) two approximations were needed. The first was to discard all nonladder graphs. The second approximation involved carrying out four-dimensional loop integrals by retaining only the positive energy proton pole contribution. The reader is referred to Ref. [10] for a more complete discussion of the procedure of obtaining the EFA. 


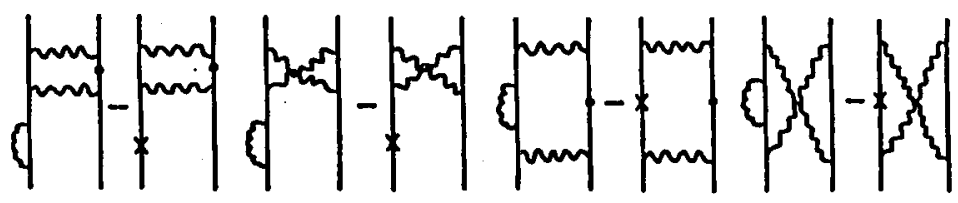

(a)

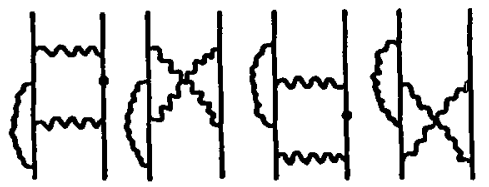

(b)

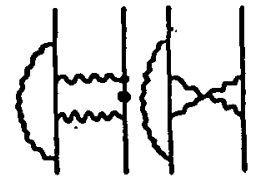

(c)

Fig. 2. Corrections to EFA. Two-photon exchange self-energy (a), vertex (b), and the spanning photons (c) diagrams. The "dot" on the proton propagator in the ladder graphs implies the subtraction of EFA, while the "cross" represents the mass renormalization.

In Ref. [10] corrections to the EFA arise from the Feynman diagrams illustrated in Fig. 2. These diagrams are complicated but they have been studied in detail and result in an additional level shift correction for the $n=2$ state of hydrogen of

$$
\frac{\alpha(Z \alpha)^{5} m^{2}}{8 M}(-0.415 \pm 0.004)
$$

which is about $-0.5 \mathrm{kIIz}$. This result was obtained by numerical integration, carried out with the program VEGAS. Since this contribution is small (less than $1 \mathrm{kIIz}$ ) we can conclude that the external field approximation is quite good and that the corrections to it, as represented by the diagrams of Fig. 2, are not essential at this time.

\section{5. $(Z \alpha)^{6} m^{2} / M$ recoil corrections}

Pure recoil corrections all originate from multiple interactions between the electron and the proton. There are numerous classes of diagrams which lead to these terms. The terms which contribute will come from double Coulomb $(\mathrm{dC})$, triple Coulomb interactions (tC), double transverse (dT), double transverse together with single Coulomb (dT-1C), and single transverse (sT). To keep track of these terms we will use for the energy shifts $\delta E_{\mathrm{ab}}^{(6)}$, where the superscript (6) denotes that these are $(\dot{Z} \alpha)^{6}$ terms and the subscripts ab characterize the origin of the terms.

\subsection{Double Coulomb}

Double Coulomb corrections are illustrated in Fig. 3. These graphs contain a leading recoil term of order $(Z \alpha)^{5} m^{2} / M$ which is calculated from $\delta V_{\mathrm{dC}}$, given by

$$
\delta V_{\mathrm{dC}}=-\int \frac{\mathrm{d}^{3} p^{\prime}}{(2 \pi)^{3}} \frac{m \gamma_{0}-E_{p^{\prime}}+\alpha \cdot p^{\prime}}{-2 E_{p^{\prime}}} \frac{-Z e^{2}}{\left(\boldsymbol{p}^{\prime}-p_{3}\right)^{2}}
$$




$$
\times \frac{-Z e^{2}}{\left(p_{1}-p^{\prime}\right)^{2}} \frac{{p^{\prime}}^{2}+p_{1} \cdot p_{3}-p^{\prime} \cdot\left(p_{1}+p_{3}\right)}{M\left(m+E_{p^{\prime}}\right)^{2}}
$$

by setting the external momenta $p_{1}$ and $p_{3}$ to zero in Eq. (8), as well as in the lower components of the external Dirac wave functions. When corrections due to the finitençss of external momenta are considered a correction $\delta E_{\mathrm{dC}}^{(6)}$ is obtained,

$$
\delta E_{\mathrm{dC}}^{(6)}=-\frac{4}{3} \frac{(Z \alpha)^{2}}{m M}\left|\psi_{2 S}(0)\right|^{2}\left(-\frac{3 \pi Z \alpha}{8}\right) .
$$

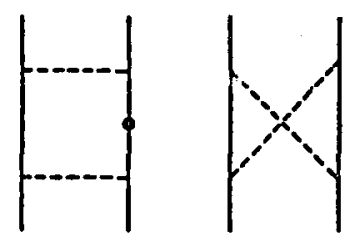

Fig. 3. Coulomb-Coulomb graphs.

\subsection{Triple Coulomb}

These terms are contained in the graphs of Fig. 4 and can be evaluated analytically (see Eq. (4.7) of Ref. [24]) by setting external momenta to zero in the graphs. It is straightforward to obtain

$$
\delta E_{\mathrm{tC}}^{(6)}=-\frac{4}{3} \frac{(Z \alpha)^{2}}{m M}\left|\psi_{2 S}(0)\right|^{2}\left(-\frac{3 \pi Z \alpha}{8}\right),
$$

which is identical to the double Coulomb piece of Eq. (9).

\subsection{Double transverse (seagull)}

The double transverse or "seagull" correction is rather subtle and consequently we will provide greater detail for this part of the calculation (see Fig. 5). The leading terms were calculated many years ago from the expression

$$
\delta V_{\mathrm{dT}}=\frac{(Z \alpha)^{2}}{m M} \int \frac{\mathrm{d}^{4} p}{\pi^{2} \mathrm{i}} \frac{(-m)\left(p_{0}-m\right) \delta_{\perp 1} \cdot \delta_{\perp 3}}{\left[\left(p-p_{3}\right)^{2}+\mathrm{i} \epsilon\right]\left[\left(p-p_{1}\right)^{2}+\mathrm{i} \epsilon\right]\left[p^{2}-m^{2}+\mathrm{i} \epsilon\right]},
$$

where $\delta_{\perp 1} \cdot \delta_{\perp 3}=1+\cos ^{2} \theta_{q_{1} q_{3}}=2-\frac{\left(q_{1} \times q_{3}\right)^{2}}{q_{1}^{2} q_{3}^{2}}$.

We make a change of variables $p_{0}-m=i \eta$ and then use

$$
\int \frac{\mathrm{d}^{4} p}{\pi^{2} \mathrm{i}}=\frac{1}{\pi^{2}} \int \mathrm{d}^{3} p \int_{-\infty}^{\infty} \mathrm{d} \eta
$$

The potential can then be expressed in terms of pieces which contribute to low-order and high-order contributions. Thus we find $\delta V_{\mathrm{dT}}=\delta V_{\mathrm{dT}}^{L 1}+\delta V_{\mathrm{dT}}^{L 2}+\delta V_{\mathrm{dT}}^{H}$, where

$$
\delta V_{\mathrm{dT}}^{L 1}=-\frac{(Z \alpha)^{2}}{m M \pi^{2}} \int \mathrm{d}^{3} p \int_{-\infty}^{\infty} \mathrm{d} \eta\left[\frac{1}{\left(\eta^{2}+q_{\mathrm{t}}^{2}\left(\eta^{2}+q_{1}^{2}\right)\right.}-\frac{1}{4 m^{2} \eta^{2}+\left(\eta^{2}+p^{2}\right)^{2}}\right],
$$




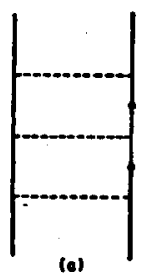

(a)

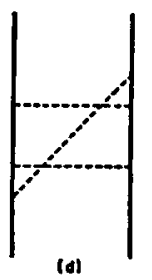

(d)

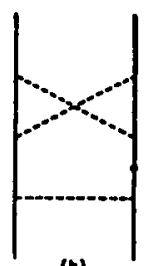

(b)

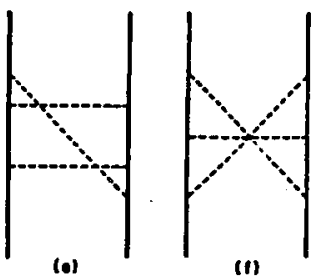

Fig. 4. Triple Coulomb diagrams.

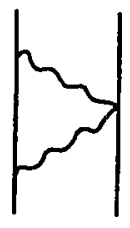

Fig. 5. Seagull correction.

$$
\begin{aligned}
\delta V_{\mathrm{dT}}^{L 2} & =\frac{(Z \alpha)^{2}}{m M \pi^{2}} \int \mathrm{d}^{3} p \int_{-\infty}^{\infty} \mathrm{d} \eta \frac{1}{\left(\eta^{2}+q_{3}^{2}\right)\left(\eta^{2}+q_{1}^{2}\right)} \frac{\left(q_{1} \times q_{3}\right)^{2}}{2 q_{1}^{2} q_{3}^{2}} \\
\delta V_{\mathrm{dT}}^{I I} & =\frac{(Z \alpha)^{2}}{m M \pi^{2}} \int \mathrm{d}^{3} p \int_{-\infty}^{\infty} \mathrm{d} \eta \frac{1}{4 m^{2} \eta^{2}+\left(\eta^{2}+p^{2}\right)^{2}} \\
\times & {\left[\frac{\left(\eta^{2}+p^{2}\right)^{2}}{\left(\eta^{2}+q_{1}^{2}\right)\left(\eta^{2}+q_{3}^{2}\right)}-1-\frac{\left(\eta^{2}+p^{2}\right)^{2}}{\left(\eta^{2}+q_{1}^{2}\right)\left(\eta^{2}+q_{3}^{2}\right)} \frac{\left(q_{1} \times q_{3}\right)^{2}}{2 q_{1}^{2} q_{3}^{2}}\right] . }
\end{aligned}
$$

These expressions must now be used with the wave functions $\phi\left(p_{1}\right)$ and $\phi\left(p_{3}\right)$ and integration over all momenta must be carried out to the desired accuracy. We have been able to implement this analytically and have found the following results for $S$ states:

$$
\begin{aligned}
& \delta E_{\mathrm{dT}}^{L 1}=\frac{2(Z \alpha)^{2}}{m M}|\psi(0)|^{2}\left[\ln Z \alpha+\left(\ln \frac{2}{n}+\sum_{i=1}^{n} \frac{1}{i}+\frac{n-1}{2 n}\right)\right], \\
& \delta E_{\mathrm{dT}}^{L 2}=\frac{2(Z \alpha)^{2}}{m M}|\psi(0)|^{2} \frac{4}{3}(1-\ln 2),
\end{aligned}
$$




$$
\delta E_{\mathrm{dT}}^{(6) H}=\frac{2(Z \alpha)^{2}}{m M}|\psi(0)|^{2}\left[-\frac{\pi Z \alpha}{2} \ln \left(\frac{2}{Z \alpha}\right)-\frac{3 \pi Z \alpha}{4}\right] .
$$

Thus for $S$ states the double transverse correction is

$$
\begin{aligned}
\delta E_{\mathrm{dT}} & =\frac{4(Z \alpha)^{2}}{3 m M}|\psi(0)|^{2} \\
\times & {\left[\frac{3}{2}\left(\ln Z \alpha+\frac{7}{4}\right)+2(1-\ln 2)-\frac{3 \pi}{4} Z \alpha \ln \left(\frac{2}{Z \alpha}\right)-\frac{9 \pi Z \alpha}{8}\right] }
\end{aligned}
$$

while for $P$ states the result is negligible.

\subsection{Double transverse-single Coulomb}

Here we evaluate the graphs shown in Fig. 6. In these diagrams a single Coulomb line corrects the seagull graph. Note that the "dot" on the proton line denotes the subtraction of those reducible graphs whose contributions are already contained in Sec. 5.3. The presence of Coulomb wave functions implies an infinite number of Coulomb ladders before and after the seagull interaction. Thus the terms must be removed to avoid double counting. We have carried out the calculation numerically. The contribution comes only from the graphs of Fig. 6c

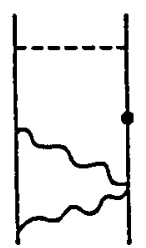

(a)

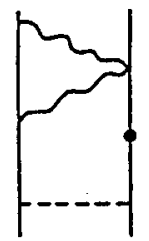

(d)

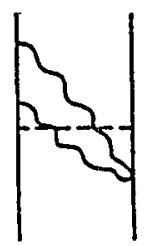

(b)

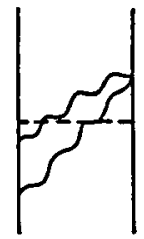

(e)

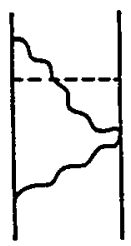

(c)

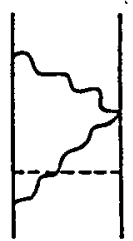

(f)

Fig. 6. Diagrams with single Coulomb and a seagull.

and Fig. 6f, that is from the graphs in which the Coulomb line is between the transverse photons. Analysis of other terms reveals a pairwise cancellation from the proton line structure whenever the Coulomb interaction precedes or follows both transverse photons. After numerical calculation we obtain

$$
\delta E_{\mathrm{dT}-1 \mathrm{C}}^{(6)}=-\frac{4(Z \alpha)^{3}}{3 m M}|\psi(0)|^{2}(-1.81) .
$$




\subsection{Single transverse}

The corrections from single transverse photons are quite complicated. Lower order terms bring in a Bethe log which signals the presence of an arbitrary number of Coulomb exchanges accompanying the single transverse exchange (See Fig. 7). To obtain the recoil effects to order $(Z \alpha)^{5} m^{2} / M$ it suffices to employ for $\Delta E_{T}$ the

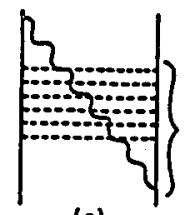

(a)

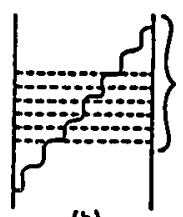

(b)

Fig. 7. Transverse exchange with multiple Coulomb exchange.

expression

$$
\Delta E_{T}=-\frac{Z \alpha}{2 \pi^{2} m M} \int \mathrm{d}^{3} k \frac{1}{k^{2}} \sum_{m} \frac{\left\langle n\left|p_{\perp} \cdot \exp (\mathrm{i} k \cdot r)\right| m\right\rangle\langle m|[p, V]| n\rangle}{E_{n}-E_{m}-k} .
$$

It is important, however, to realize that this approximation is based on old-fashioned perturbation theory and moreover the intermediate states only include positive energy states. Thus we will need corrections from negative energy states. We return to this later.

The expression (17) above readily yields $(Z \alpha)^{5} \mathrm{~m}^{2} / M$ terms but also contains a $(Z \alpha)^{6} m^{2} / M$ piece. We have handled this by writing

$$
\begin{gathered}
\frac{\exp (\mathrm{i} k \cdot r)}{E_{n}-E_{m}-k}=\frac{1}{E_{n}-E_{m}-k}+\frac{\exp (\mathrm{i} k \cdot r)-1}{-k}+\frac{[\exp (\mathrm{i} k \cdot r)-1]\left(E_{n}-E_{m}\right)}{k\left(E_{n}-E_{m}-k\right)} \\
\equiv T_{1}+T_{2}+T_{2}^{\prime} .
\end{gathered}
$$

$T_{1}$ and $T_{2}$ terms will produce the $(Z \alpha)^{5} \mathrm{~m}^{2} / M$ piece while $T_{2}^{\prime}$. will produce a higher order correction. This higher order correction is easy to calculate and we obtain

$$
\delta E_{T_{2}^{\prime}}^{(6)}=-\frac{4}{3} \frac{(Z \alpha)^{2}}{m M}|\psi(0)|^{2}\left(-\frac{9 \pi}{4} Z \alpha\right) .
$$

Now let us turn to additional corrections which are not properly contained in the single particle theory. There are two distinct single loop contribution to the energy shift would be

$$
\begin{aligned}
& \Delta E_{\mathrm{C}-\mathrm{T}}=\frac{8(Z \alpha)^{2}}{(2 \pi)^{7} M} \int \mathrm{d}^{3} k \mathrm{~d}^{3} p_{i} \mathrm{~d}^{3} p_{f} \frac{1}{2 E_{p_{i}-k}\left(p_{i}-p_{f}-k\right)^{2}} \phi^{\dagger}\left(p_{f}\right) \\
& \times \frac{1}{2 k^{2}}\left\{\frac{E_{p_{i}-k}+\alpha \cdot\left(p_{i}-k\right)+\beta m}{k-p_{i o}+E_{p_{i}-k}}+\frac{E_{p_{i}-k}-\alpha \cdot\left(p_{i}-k\right)-\beta m}{k-p_{i o}-E_{p_{i}-k}}\right. \\
& \left.\times\left[1-\frac{2 k^{2}}{\left(p_{i o}+E_{p_{i}-k}\right)\left(p_{i_{0}}+E_{p_{i}-k}+k\right)}\right]\right\} \times \alpha_{\perp} \cdot\left(p_{i}-p_{f}\right) \phi\left(p_{i}\right) .
\end{aligned}
$$

contributions, the first coming from single loop Feynman diagrams containing one Coulomb and one transverse (see Fig. 8), and the second coming from two-loop 

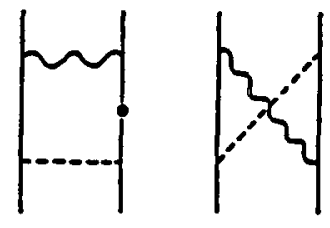

(a)
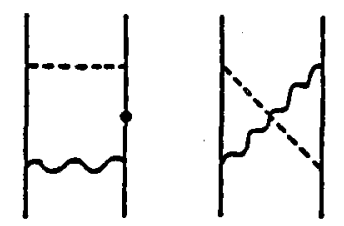

(b)

Fig. 8. Single Coulomb-single transverse graphs.

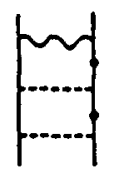

(a)

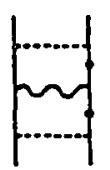

(a)

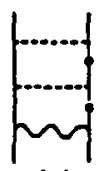

(m)

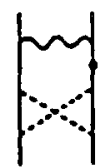

(b)

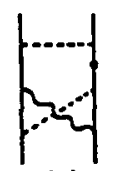

(n)

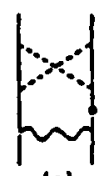

(n)

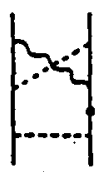

(c)

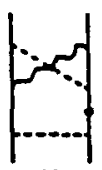

(i)

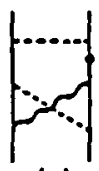

(1)

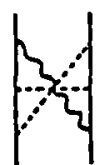

(d)

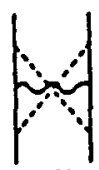

(i)

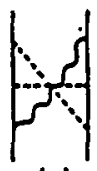

(o)

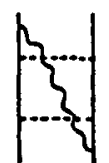

(e)

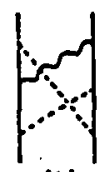

(I)

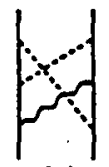

(a)

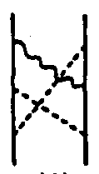

(II)

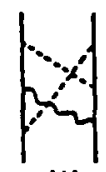

(I)

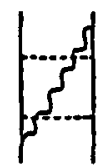

(r)

Fig. 9. Double Coulomb-single transverse graphs.

graphs with two Coulombs and one transverse (see Fig. 9). The expression for the To obtain this expression we decomposed the electron propagator into positive and negative energy terms and then carried out the part of the loop integral $\left(\int \mathrm{d}^{4} k\right)$ by integrating over $k_{0}$. The first term arises from a pole at $k_{0}=k-\mathrm{i} \epsilon$ and so does the second. The third term comes from the negative energy electron pole at $k_{0}=p_{i o}+E_{p_{i}-k}$. We need to remove from the above expression those terms which have already been calculated in the single particle theory approximation. The entire approximation is given by (5.18) of Ref. [24] and the piece to be removed is obtained by using the leading approximation to the nonrelativistic propagator. Thus we subtract $\Delta E_{+}$, where

$$
\Delta E_{+}=\frac{8(Z \alpha)^{2}}{(2 \pi)^{7} m M} \int \mathrm{d}^{3} k \mathrm{~d}^{3} p_{i} \mathrm{~d}^{3} p_{f} \frac{1}{k^{2}\left(p_{i}-p_{f}-k\right)^{2}} \tilde{\phi}^{\dagger}\left(p_{f}\right)
$$




$$
\times \frac{p_{i \perp} \cdot\left(p_{i}-p_{f}\right)}{2 m k+\gamma^{2}+\left(p_{i}-k\right)^{2}} \tilde{\phi}\left(p_{i}\right) .
$$

We have numerically computed $\Delta E_{\mathrm{C}-\mathrm{T}}-\Delta E_{+}$for $S$ and $P$ states. The $P$ state contributions are negligible and we obtain a result of $8.02 \mathrm{kHz}$ for $n=2$. The bulk of this comes from the negative energy projection piece of Eq. (20) while the remainder is the difference between the positive energy piece and $\Delta E_{+}$(its approximation). A good portion of this correction can be extracted analytically to produce $\ln (1 / Z \alpha)$. We present the result in this form and provide a correction which is obtained numerically. Thus we have

$$
\delta E_{\mathrm{C}-\mathrm{T}}^{(6)}-\delta E_{+}^{(6)}=\frac{-4(Z \alpha)^{3}|\psi(0)|^{2}}{3 m M}\left[-\frac{3 \pi}{2} \ln \left(\frac{1}{Z \alpha}\right)+8.30(5)\right] .
$$

Next we turn to the last contribution from Fig. 9 which involves a transverse photon with two Coulomb interactions. A number of diagrams are already accounted for and double counting must be avoided. Thus all diagrams in which the transverse photon precedes or follows the Coulomb interactions must be discarded. In addition, according to Sapirstein and Yennie [29] diagrams in which the electron line has a transverse between two Coulombs are suppressed relative to leading terms. Ultimately, after removing unimportant terms and setting the

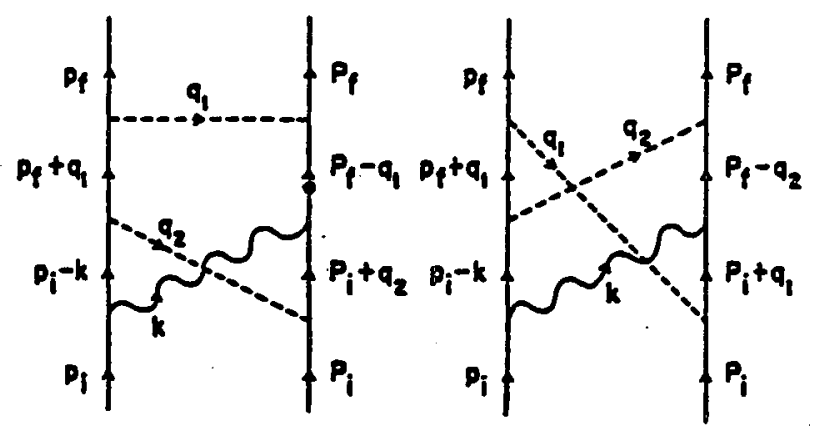

Fig. 10. Contributing double Coulomb-single transverse graphs.

external momenta to zero, the contributions come only from Fig. 10 and we obtain a manageable expression which we have integrated numerically. We find an $n=2$ effect of $-1.9 \mathrm{kHz}$. Thus we can write

$$
\delta E_{\mathrm{dC}-\mathrm{T}}^{(6)}=-\frac{4(Z \alpha)^{3}|\psi(0)|^{2}}{3 m M}(3.53) \text {. }
$$

This completes the contributions from a transverse photon to order $(Z \alpha)^{6} m^{2} / M$. The total result of this order is

$$
(Z \alpha)^{6} \frac{m^{2}}{M n^{3}}\left[\frac{5}{2}+\ln \left(\frac{1}{2 Z \alpha}\right)-4.25\right] \text {. }
$$




\section{Corrections of order $\alpha^{2}(Z \alpha)^{5} m$}

Corrections to the Lamb shift of order $\alpha^{2}(Z \alpha)^{5} \mathrm{~m}$ arise from six classes of diagrams shown in the graphs of Figs. 11a-f. These graphs arise from a single skeleton diagram, Fig. 12, involving two Coulomb interactions. Thus far, results have been obtained for Figs. 11a-d. We will remind the reader of these contributions which have been published elsewhere [25-27].

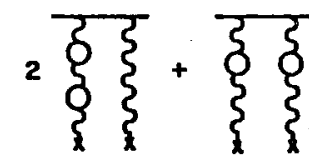

a

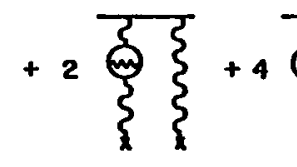

b

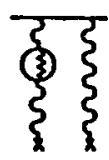

$+2$
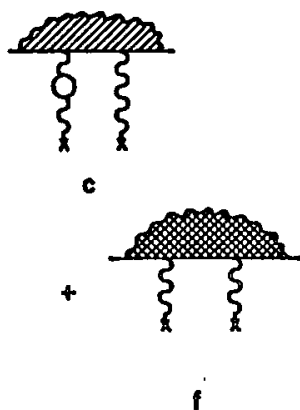

Fig. 11. All gauge invariant sets of diagrams that generate corrections of order $\alpha^{2}(Z \alpha)^{5} m$ to the Lamb shift.

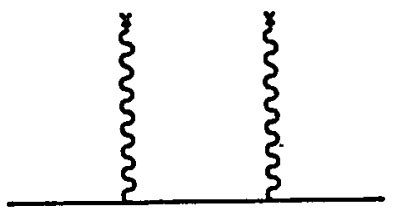

Fig. 12. Skeleton graph.

The contribution from Fig. 11a comes from the several lowest order vacuum polarization insertions on the skeleton graph. This produces the simple result

$$
\Delta E_{a}=\Delta E_{1 p}=-\frac{48}{n^{3}} m\left(\frac{\mu_{r}}{m}\right)^{3}\left(\frac{\alpha}{\pi}\right)^{2} \frac{(Z \alpha)^{5}}{\pi} \int_{0}^{\infty} \mathrm{d} k I_{1}^{2}(k),
$$

where

$$
I_{1}(k)=\int_{0}^{1} \mathrm{~d} v \frac{v^{2}\left(1-v^{2} / 3\right)}{4+\left(1-v^{2}\right) k^{2}}
$$

The integrations are straightforward to perform numerically and they yield the result

$$
\Delta E_{a}=-0.0609(1) \frac{1}{n^{3}}\left(\frac{\mu_{r}}{m}\right)^{3} \frac{\alpha^{2}(Z \alpha)^{5}}{\pi} m \text {. }
$$

Next consider the irreducible two-loop vacuum polarization pictured in Fig. 11b. Here the calculation leads to the expression

$$
\Delta E_{b}=\Delta E_{2 p}=-\frac{32}{n^{3}} m\left(\frac{\mu_{r}}{m}\right)^{3}\left(\frac{\alpha}{\pi}\right)^{2} \frac{(Z \alpha)}{\star} \int_{0}^{\infty} \frac{\mathrm{d} k}{k^{2}} \tilde{I}_{2}(k),
$$


where

$$
\tilde{I}_{2}(k)=I_{2}(k)-I_{2}(0)
$$

with $I_{2}(k)$ the two-loop polarization operator. This is given by

$$
\begin{aligned}
I_{2}(k) & =\frac{2}{3} \int_{0}^{1} \mathrm{~d} v \frac{v}{4+\left(1-v^{2}\right) k^{2}}\left\{( 3 - v ^ { 2 } ) ( 1 + v ^ { 2 } ) \left[\operatorname{Li}\left(-\frac{1-v}{1+v}\right)\right.\right. \\
+ & \left.2 \operatorname{Li}\left(\frac{1-v}{1+v}\right)+\frac{3}{2} \ln \frac{1+v}{1-v} \ln \frac{1}{2}(1+v)-\ln \frac{1+v}{1-v} \ln v\right] \\
+ & {\left[\frac{11}{16}\left(3-v^{2}\right)\left(1+v^{2}\right)+\frac{1}{4} v^{4}\right] \ln \frac{1+v}{1-v} } \\
+ & {\left.\left[\frac{3}{2} v\left(3-v^{2}\right) \ln \frac{1}{4}\left(1-v^{2}\right)-2 v\left(3-v^{2}\right) \ln v\right]+\frac{3}{8} v\left(5-3 v^{2}\right)\right\}, }
\end{aligned}
$$

where $\operatorname{Li}(z)$ is the Euler dilogarithm (see e.g., Refs. [30], [31]).

The subtraction of $I_{2}(0)$ is necessary because without this we encounter a low $k$ divergence indicative of a contribution of lower order. This lower order term was obtained many years ago [16] and must be removed. After this is done the integration of Eq. (28) may readily be carried out numerically. The result obtained is

$$
\Delta E_{b}=0.5080(1) \frac{1}{n^{3}}\left(\frac{\mu_{r}}{m}\right)^{3} \frac{\alpha^{2}(Z \alpha)^{5}}{\pi} m .
$$

A third correction, from Fig. 11c involves the presence of a vacuum polarization inserted on the lowest order radiative electron factor analyzed in Ref. [10]. This correction is

$$
\Delta E_{c}=-\frac{32}{n^{3}} m\left(\frac{\mu_{r}}{m}\right)^{3}\left(\frac{\alpha}{\pi}\right)^{2} \frac{(Z \alpha)^{5}}{\pi} \int_{0}^{\infty} \mathrm{d} k I_{1}(k) L(k),
$$

where $I_{1}(k)$ is the lowest order vacuum polarization expression given earlier while $L(k)$ is the electron factor given in Ref. [10]

$$
\begin{aligned}
L(k)= & -\frac{1}{8} \int_{0}^{1} \int_{0}^{1} \mathrm{~d} x \mathrm{~d} y \\
& \times\left[\frac{A_{3}}{\Delta^{3}}+\frac{A_{2}}{\Delta^{2}}+\frac{A_{1}}{\Delta}+\frac{B_{2}}{\Delta^{2} D_{0}}+\frac{B_{1}}{\Delta D_{0}}+\frac{E_{0}}{D_{0}}+\frac{E_{1}}{\Delta_{1} D_{0}}\right] .
\end{aligned}
$$

The various terms are defined in Ref. [10]. The integrations required are nontrivial since we must eliminate low $k$ singular behavior by means of analytic rather than numerical cancellations. Thus the terms involving $B_{2}, B_{1}, E_{0}$, and $E_{1}$, all of which contain $1 / k^{2}$ behavior from $D_{0}$, require special treatment. We have done this, as reported in Ref. [10], and were then able to do the numerical integration. The result then obtained from Eq. (32) is

$$
\Delta E_{c}=0.611(1) \frac{1}{n^{3}}\left(\frac{\mu_{r}}{m}\right)^{3} \frac{\alpha^{2}(Z \alpha)^{5}}{\pi} m
$$

To check our calculation, which was performed in the Fried-Yennie gauge, we also calculated the electron factor in the Feynman gauge and repeated the calculation of $\Delta E_{c}$, obtaining an exact confirmation of the previous result. We also checked the asymptotic limits of $L(k)$ and confirmed their agreement with accepted results. 
A fourth correction of order $\alpha^{2}(Z \alpha)^{5} \mathrm{~m}$ arises from the diagrams illustrated in Fig. 11d. This set of graphs requires a vacuum polarization insertion on the radiative photon and thus appears as a correction to the electron factor diagrams studied in Ref. [10]. Unfortunately, the expression for $L(k)$ was given in Ref. [10] in the Fried-Yennie gauge and was infrared regularized by using off-shell electrons rather than by introducing a photon mass $\lambda$. It would have been useful to have the latter expression since the inclusion of vacuum polarization could readily be accomplished through integration over a variable mass $\lambda(v)$.

The approach we adopted was to recalculate the expression corresponding to $L(k)$ in the Feynman gauge of the radiative photon of mass $\lambda(v)$ and then to numerically integrate over $v$. This is reported in Ref. [27] where we find that

$$
\Delta E_{d}=\frac{-16}{n^{3}} m\left(\frac{\mu_{r}}{m}\right)^{3}\left(\frac{\alpha}{\pi}\right)^{2} \frac{(Z \alpha)^{5}}{\pi} \int_{0}^{\infty} \mathrm{d} k \frac{\mathcal{L}(k)-\mathcal{L}(0)}{k^{2}}
$$

with

$$
\mathcal{L}(k)=\int_{0}^{1} \mathrm{~d} v \frac{v^{2}\left(1-v^{2} / 3\right)}{1-v^{2}} L(k, \lambda) .
$$

In this expression the photon mass $\lambda$ is given by

$$
\lambda^{2}=4 /\left(1-v^{2}\right) \text {. }
$$

The electron factor $L(k, \lambda)$ is the Feynman gauge expression, which is given by Eqs. $(3,4)$ of Ref. [27].

Once again we note that subtraction at zero momentum is carried out. This renders the $k$ integral infrared finite and removes a contribution of lower order. The resulting expression may be integrated to obtain

$$
\Delta E_{\mathrm{d}}=-0.0729(1) \frac{1}{n^{3}}\left(\frac{\mu_{r}}{m}\right)^{3} \frac{\alpha^{2}(Z \alpha)^{5}}{\pi} m
$$

The additional contributions from Figs. 11e, f have not been fully evaluated. The first of these, (e), comes from light by light scattering while the second, (f), arises from two radiative photons on the electron line. Work is now in progress on these difficult terms.

\section{Conclusions}

Currently, the theoretical splitting of the $2 S_{\frac{1}{2}}-2 P_{\frac{1}{2}}$ levels disagrees with the experimental result. This is apparent from the results presented in Secs. 2 and 3. It is likely that the theoretical expression is incomplete and that further research must be carried out.

There are clearly uncalculated corrections of order $\alpha^{2}(Z \alpha)^{5} m$ which arise from two classes of Feynman diagrams. The first class, shown in Fig. 11e, consists of three-loop graphs which contain virtual light by light scattering. The second class, illustrated schematically in Fig. 11f, contains all possible two-photon insertions on the electron line. These are also three-loop graphs. These classes of graphs can be evaluated by setting the external momenta to zero. Research on both of the above sets of diagrams is now in progress. In addition, it is also necessary to calculate any corrections of order $\alpha^{3}(Z \alpha)^{4} m$ which might arise from three photons on the 
electron line. These would come from the contribution to the slope of the Dirac form factor resulting from three photon corrections to the vertex.

In the theoretical expression presented in Sec. 2 there is an important correction due to the radius of the proton. From a theorist's point of view this is the simplest theoretical contribution to the Lamb shift. In effect the extension of the proton shifts the $S$ states but not the $P$ states since the former states have a finite wave function at the origin while the latter have a vanishing wave function. The difficulty which arises is that the proton radius cannot be reliably calculated theoretically and therefore the theory relies on an experimental determination of this radius, which is defined as

$$
\left\langle r_{\mathrm{p}}^{2}\right\rangle=\int \mathrm{d}^{3} r_{N} \rho\left(r_{N}\right) r_{N}^{2},
$$

where $\rho\left(R_{N}\right)$ is the nuclear charge density. For hydrogen $\rho$ is the proton charge density which is obtained from elastic electron proton scattering.

The two most credible values of this radius $r_{\mathrm{p}}, 0.805(11) \mathrm{fm} \mathrm{[20]}$ and 0.862 (12) $\mathrm{fm}$ [19], disagree with each other and produce an $18 \mathrm{kHz}$ difference for the hydrogen Lamb shift. The smaller value gives a result which is closer to experiment than does the larger value. It might be argued that the larger value should be used. The experiment is more recent and consequently the authors of Ref. [19] had the benefit of prior knowledge and possible sources of error in preceding experiments. The authors of Ref. [20] assumed a definite form for the charge distribution by using specific form factors. In contrast to this, the authors of Ref. [19] did not presume any form to determine the value of the radius. In either case, however, it does appear that the slope of the form factor at low $q^{2}$ is measured and it is difficult to assert with confidence that one result is more reliable than the other. Needless to say, it would be highly desirable to have a new and more accurate determination of the proton radius. This is clearly needed to reduce the uncertainty in the theory of the hydrogen Lamb shift.

\section{Acknowledgment}

I am deeply grateful for the support of the National Science Foundation which has funded this research. The present support is under the grant number PIIY- 9120102. Over the years I have greatly benefited from conversation and/or collaboration with G. Bhatt, M. Doncheski, D.A. Owen, D.R. Yennie, P.J. Mohr, G.W. Erickson, M.I. Eides and E. Kazes on many aspects of the hydrogen Lamb shift.

\section{References}

[1] W.E. Lamb, Jr., R.C. Retherford, Phys. Rev. 72, 241 (1947).

[2] H.A. Bethe, Phys. Rev. 72, 239 (1947).

[3] J. Schwinger, V. Weisskopf, Phys. Rev. 73, 1272A (1948).

[4] J.R. Oppenheimer, Phys. Rev. 35, 461 (1930).

[5] S.R. Lundeen, F.M. Pipkin, Phys. Rev. Lett. 46, 232 (1981); Metrologia 22, 9 (1986). 
[6] Yu.L. Sokolov, U.P. Yakovlev, Zh. Eksp. Teor. Fiz. 83, 15 (1982); V.G. Palchikov, Yu.L. Sokolov, V.P. Yakovlev, Pisma Zh. Eksp. Teor. Fiz. 38, 347 (1983) [JETP Lett. 38, 418 (1983)].

[7] see P.J. Molir, Phys. Rev. A 46, 4421 (1992) for recent theoretical results.

[8] G.W.F. Drake, R.B. Grimley, Phys. Rev. A 8, 157 (1973); A. van Wijngaarden, G.W.F. Drake, P.S. Farago, Phys. Rev. Lett. 33, 4 (1974); G.W.F. Drake, S.P. Goldman, A. van Wijngaarden, Phys. Rev. A 20, 1299 (1979); J. Patel, A. van Wijngaarden, G.W.F. Drake, Phys. Rev. A 36, 5230 (1987); G.W.F. Drake, J. Patel, A. van Wijngaarden, Phys. Rev. Lett. 60, 1002 (1988). See also Ref. [14] of the present paper.

[9] Leading radiative corrections to bound state levels were given in many papers. H.A. Bethe, Phys. Rev. 72, 339 (1947); F.J. Dyson, Phys. Rev. 73, 617 (1948); H. Fukuda, Y. Miyamoto, S. Tomonaga, Progr. Theoret. Phys. (Kyoto) 4, 47,121 (1949); N.M. Kroll, W.E. Lamb, Jr., Phys. Rev. 75, 388 (1949); J. Schwinger, Phys. Rev. 75, 989 (1949); J.B. French, V.F. Weisskopf, Phys. Rev. 75, 1240 (1949); R.P. Feynman, Phys. Rev. 76, 769 (1949).

[10] G. Bhatt, H. Grotch, Phys. Rev. A 31, 2794 (1985); Phys. Rev. Lett. 58, 471 (1981); Ann. Phys. 178, 1 (1987).

[11] R. Karplus, A. Klcin, J. Schwinger, Phys. Rev. 86, 288 (1952); M. Baranger, H.A. Bethe, R.P. Feynman, Phys. Rev. 92, 482 (1953); G.W. Erickson, D.R. Yennie, Ann. Phys. (NY) 35, 271 (1965); 35, 447 (1965).

[12] A.J. Layzer, Phys. Rev. Lett. 4, 580 (1960); H.M. Fried, D.R. Yennie, Phys. Rev. Lelt. 4, 583 (1960); A.J. Layzer, J. Math. Phys. 2, 292 (1961).

[13] G.W. Erickson, Phys. Rev. Lett. 27, 780 (1971); P.J. Mohr, Phys. Rev. Lett. 34, 1050 (1975); J.R. Sapirstein, Phys. Rev. Lett. 47, 1723 (1981).

[14] A. van Wijngaarden, J. Kwela, G.W.F. Drake, Phys. Rev. A 43, 3325 (1991).

[15] K. Pachucki, Phys. Rev. A 46, 648 (1992).

[16] M. Baranger, F. Dyson, E.E. Salpeter, Phys. Rev. 88, 680 (1952); J. Weneser, R. Bersohn, N.M. Kroll, Phys. Rev. 91, 1257 (1953); C.M. Sommerfield, Phys. Rev. 107, 328 (1957); T. Appelquist, S.J. Brodsky, Phys. Rev. B 2, 2293 (1970).

[17] E.E. Salpeter, Phys. Rev. 87, 328 (1952); T. Fulton, P.C. Martin, Phys. Rev. 95, 811 (1954).

[18] See Ref. [11], Erickson and Yennie.

[19] G.G. Simon, Ch. Schmitt, F. Borkowski, V.H. Walther, Nucl. Phys. A 333, 381 (1981).

[20] L.N. Hand, D.J. Miller, R. Wilson, Rev. Mod. Phys. 35, 335 (1963).

[21] W.A. Barker, F.N. Glover, Phys. Rev. 99, 317 (1955).

[22] D.A. Owen, Ann. Phys. Fr. 11, 249 (1986).

[23] G.W. Erickson, H. Grotch, Phys. Rev. Lett. 60, 2611 (1988); 63, 1326 (1989);

M.A. Doncheski, H. Grotch, D.A. Owen, Phys. Rev. A 41, 2851 (1990).

[24] M. Doncheski, H. Grotch, G.W. Erickson, Phys. Rev. A 43, 2152 (1991).

[25] M.I. Eides, H. Grotch, D.A. Owen, Phys. Letts. B 294, 115 (1992).

[26] M.I. Eides, H. Grotch, Phys. Letts. B 301, 127 (1993).

[27] M.I. Eides, H. Grotch, Phys. Letts. B 308, 389 (1993).

[28] H. Grotch, D.R. Yennie, Rev. Mod. Phys. 41, 350 (1969). 
[29] J. Sapirstein, D.R. Yennie, in: Quantum Electrodynamics, Ed. T. Kinoshita, World Scientific, Singapore 1990, p. 560.

[30] J. Schwinger, Particles, Sources and Fields, Vol. 2, Addison-Wesley, Reading (MA) 1973.

[31] S.G. Karshenboim, V.A. Shelyuto, M.I. Eides, Sov. Phys. JETP 65, 664 (1987). 